\title{
Efeito agudo do alongamento estático sobre a força muscular dinâmica no exercício supino reto realizado em dois diferentes ângulos articulares
}

\author{
Acute effect of static stretching on dynamic muscular strength on bench \\ press exercise at two different joint angles
}

\author{
Eurico Peixoto César ${ }^{1}, 2$, César Augusto P Paula², Denise Paulino², Luciana Miranda Lima \\ Teixeira² ${ }^{2}$ Paulo Sérgio Chagas Gomes ${ }^{1 *}$
}

ARTIGO ORIGINAL | ORIGINAL ARTICLE

\begin{abstract}
O objetivo foi determinar os efeitos agudos do alongamento estático (AL) sobre os níveis de amplitude de movimento (ADM) da cintura escapular e sobre 10 repetições máximas (10-RM) no supino reto em dois ângulos articulares. Onze homens $(28.32 \pm 5.4$ anos; $1.73 \pm 0.1 \mathrm{~m} ; 72.14 \pm 15.3 \mathrm{~kg})$ foram submetidos aleatoriamente à condição controle (CC) e experimental (2 séries de $30 \mathrm{~s}$ de AL passivos para o peitoral e tríceps). A ADM da cintura escapular no movimento de abdução horizontal do ombro foi analisada através da fotogrametria usando ferramenta de dimensão angular do CorelDRAW ${ }^{\circledR}$. Os testes de 10-RM foram feitos no supino reto com barra livre com randomização para amplitude total e limitada a $90^{\circ}$ de flexão do cotovelo. A confiabilidade da ADM da cintura escapular e das 10-RM nas duas amplitudes diferentes foi alta $(\mathrm{R}>0.95)$. A ANOVA de duas entradas com medidas repetidas nos dois fatores com post-hoc de Bonferroni indicou uma interação significativa, com aumento da ADM ( $p=$ 0.029) após AL. Não foi encontrada diferença significativa para 10-RM e volume total em nenhuma das angulações e condições testadas. Conclui-se que volumes reduzidos de AL aumentaram significativamente a ADM sem promover prejuízo no desempenho da força dinâmica realizada em diferentes ângulos articulares.
\end{abstract}

Palavras-chave: amplitude de movimento articular, avaliação do desempenho, treinamento de resistência.

\begin{abstract}
The objective of the study was to determine the acute effects of static stretching (SS) on the range of motion (ROM) levels of the shoulder girdle and on 10 repetition maximum (10-RM) on the bench press exercise in two joint angles. Eleven male subjects $(28.32 \pm 5.4$ years; $1.73 \pm 0.1 \mathrm{~m} ; 72.1 \pm 15.3 \mathrm{~kg})$ were randomly assigned to a control (CC - no stretching) and experimental (two sets of $30 \mathrm{~s}$ of passive static stretching for the chest and the triceps) conditions. The shoulder girdle ROM during horizontal abduction of the shoulder was analyzed measure with photogrammetry and analyzed using an angular dimension tool from CorelDRAW®. The 10-RM was performed in a free bar bench press in two different joint angles (total range of motion or $90^{\circ}$ of elbow flexion). The reliability of shoulder girdle ROM and $10-\mathrm{RM}$ at $90^{\circ}$ and total range were high $(\mathrm{R}>0.95)$. A two-way ANOVA with repeated measures for both factors with post-hoc Bonferroni test showed a significant interaction with increase in ROM ( $p$ 0.029) after SS. No significant differences were found for the others variables. It was concluded that a reduced static stretching routine was able to increase acute ROM levels without impairing dynamic strength performance at different joint angles.
\end{abstract}

Keywords: range of motion, employee performance appraisal, resistance training

\footnotetext{
Artigo recebido a 15.08.2013; Aceite a 12.09.2014

${ }^{1}$ Laboratório Crossbridges, Programa de Pós-graduação em Ciências do Exercício e do Esporte - PPGCEE, Instituto de Educação Física e Desportos, Universidade do Estado do Rio de Janeiro - UERJ

${ }^{2}$ Curso de Educação Física, Universidade Presidente Antônio Carlos (Barbacena, MG)

* Autor correspondente: PPGCEE, UERJ, Rua São Francisco Xavier, No. 524, Pavilhão João Lyra Filho - Bloco $\mathrm{F}-9^{0}$ andar, Sala 9122. Maracanã - Rio de Janeiro - RJ - 20550-900, Brasil,

E-mail: gomespscg@yahoo.com.br
} 


\section{INTRODUÇÃO}

Apesar de o alongamento estático ser frequentemente utilizado durante a rotina de aquecimento em diversas modalidades desportivas, o efeito dessa estratégia no desempenho da força e potência tem sido amplamente debatido na literatura, com alguns autores apontando prejuízo na atividade subsequente (Bacurau et al., 2009; Bradley, Olsen, \& Portas, 2007; Ogura, Miyahara, Naito, Katamoto, \& Aoki, 2007), enquanto outros não foram capazes de encontrar efeitos deletérios sobre o desempenho (Beedle, Rytter, Healy, \& Ward, 2008; Perrier, Pavol, \& Hoffman, 2011). O leitor deve referir-se à revisão de Rubini, Costa, \& Gomes (2007) para maiores considerações sobre o assunto.

A discordância entre esses achados parece estar associada aos diferentes volumes e/ou o tempo de insistência do alongamento estático empregados. Tal questão tem sido investigada na literatura e apontada como a possível relação dose-reposta dos efeitos do alongamento estático sobre o desempenho (Ryan et al., 2008). Além disso, alguns trabalhos que utilizaram volumes menores de alongamento estático apresentaram aumento na amplitude de movimento (ADM) articular sem prejuízo no desempenho da força (Ogura et al., 2007; Ryan et al., 2008), o que reforça a importância da manipulação adequada do volume do alongamento para o efeito desejado.

Atualmente tem sido levantada a hipótese dos efeitos do alongamento estático ser ânguloespecífico, com alguns autores evidenciando queda no desempenho em algumas angulações articulares, mas não em outras (La Torre et al., 2010; Nelson, Guillory, Cornwell, \& Kokkonen, 2001; Weir, Tingley, \& Elder, 2005). O mecanismo postulado para explicar tal fenômeno seria a redução da rigidez passiva da unidade músculo-tendinosa, o que provocaria uma alteração para a direita na curva de comprimento tensão (Weir et al., 2005), obrigando o músculo a trabalhar em amplitudes de movimento maiores após alongamento estático para obter os mesmos níveis de tensão máxima alcançados sem a rotina de alongamentos. No entanto, até onde se sabe tal hipótese só foi testada em exercícios para membros inferiores, sem relatos na literatura sobre o possível efeito do alongamento estático sobre a curva de comprimento-tensão em músculos do membro superior.

Diante das divergências e lacunas apontadas acima, o presente estudo objetivou determinar os efeitos agudos de uma rotina de alongamentos estáticos sobre os níveis de amplitude de movimento da cintura escapular e sobre a força de resistência de membros superiores no exercício supino reto com amplitude de movimento total ou limitada a $90^{\circ}$ de flexão do cotovelo.

\section{MÉTODOS}

O presente estudo, com delineamento de grupo único com tratamento cruzado aleatório, foi aprovado pelo Comitê de Ética em Pesquisa da Universidade Presidente Antônio Carlos UNIPAC - MG ( ${ }^{\circ}$ 171.510/2012). O envolvimento dos voluntários somente ocorreu após a assinatura do termo de consentimento livre e esclarecido, além do preenchimento de um questionário para estratificação de risco e uma explicação detalhada dos procedimentos do presente estudo. O estudo foi conduzido de acordo com os preceitos definidos na declaração de Helsínquia para pesquisas com seres humanos.

\section{Amostra}

A amostra foi composta por 11 voluntários do sexo masculino $(28.32 \pm 5.4$ anos; $1.73 \pm$ $0.1 \mathrm{~m} ; 72.14 \pm 15.3 \mathrm{~kg}$ ), estudantes de educação física, fisicamente ativos e com experiência de pelo menos dois anos no exercício supino reto na barra livre. Nenhum dos sujeitos fazia uso de suplementos alimentares e esteroides anabólicos ou apresentou lesão na cintura escapular ou cotovelo nos seis meses que antecederam a coleta de dados.

\section{Instrumentos}

No presente estudo foi medido o nível de ADM da cintura escapular no movimento de AHO com o cotovelo estendido e palma da mão voltada para baixo. Inicialmente foram marcados os pontos anatômicos acromial 
(borda superior e lateral do processo acromial) e estiloidal (ponto mais distal do processo estiloide do rádio) no membro superior direito dos voluntários.

Em seguida o sujeito ficou deitado em um banco em decúbito ventral, com o quadril e o tronco fortemente presos através de faixas, garantindo assim estabilização do tronco e cintura escapular durante a medida. A partir daí, com o ombro abduzido a $90^{\circ}$, o cotovelo estendido e palma da mão voltada para baixo, foi feita uma mobilização lenta e gradual com o movimento de $\mathrm{AHO}$ até o ponto de maior desconforto relatado pelo sujeito (Figura 1).

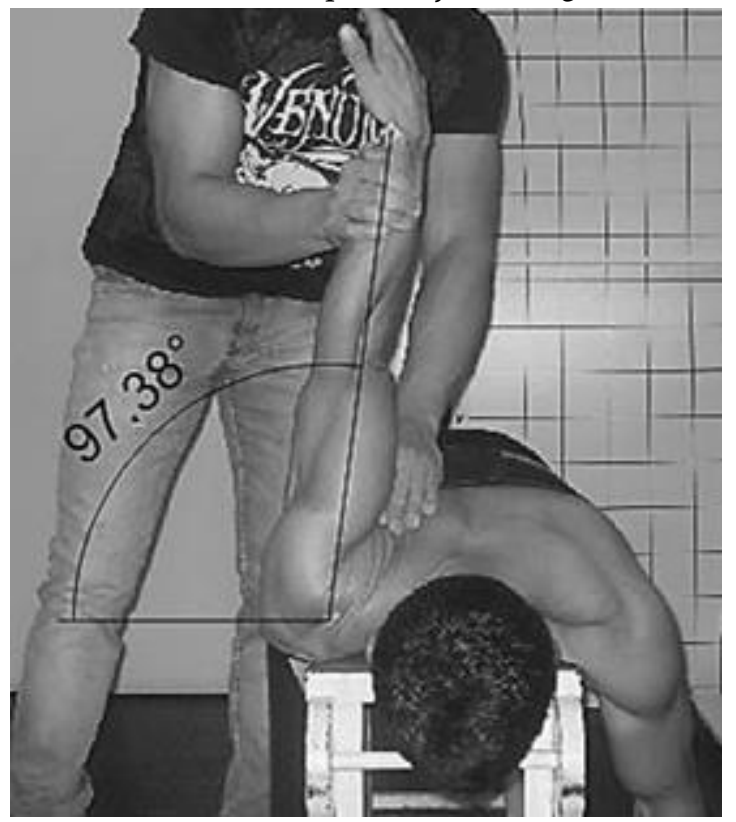

Figura 1. Análise da ADM da cintura escapular no movimento de abdução horizontal da articulação do ombro com o método de fotogrametria

Identificado esse ponto, a postura foi sustentada por dois segundos para o registro da medida através de fotografia (Sony Cyber-shot DSC-H5, San Diego, USA) com frequência de amostragem de $30 \mathrm{~Hz}$ posicionada em um plano horizontal fixada em um tripé situada em uma plataforma de $80 \mathrm{~cm}$ de altura a uma distância de $1.60 \mathrm{~m}$ do banco com o quadro centralizado na articulação do ombro do voluntário testado. Foram realizados três movimentos e utilizou-se a média entre as medidas para análise.

Para se determinar a ADM foi empregado o método de fotogrametria utilizando a ferramenta dimensão angular, disponível no software CorelDRAW ${ }^{\circledR}$ Graphics Suite - Versão
12.0. A confiabilidade desse método foi determinada através das medidas realizadas durante as visitas dois e três do estudo.

Para se determinar a força de resistência dinâmica máxima dos sujeitos foi empregado o teste de 10-RM no exercício supino reto com barra livre (barra cromada de $180 \mathrm{~cm}$, Terra Fitness, Santo André, São Paulo, Brasil). Antes do início dos testes, todo o procedimento foi explicado detalhadamente aos sujeitos.

Inicialmente foi solicitado que o sujeito utilizasse a pegada na barra que lhe fosse mais confortável sendo essa padronizada através de marcações para as demais visitas. No teste com limitação de $\mathrm{ADM}$ a $90^{\circ}$ de flexão do cotovelo, foi utilizado um aparato de metal com medidas de ajuste de altura limitadas por elásticos posicionados em ambos os lados do banco de supino. Para determinação da ADM, foi solicitado ao sujeito que descesse controladamente a barra sem nenhuma carga até que o ângulo entre o seu braço e antebraço formassem $90^{\circ}$. Tal medida foi feita através de um goniômetro universal. Nesse ponto as tiras elásticas do aparato foram posicionadas, tocando o braço do sujeito e foi anotada a medida para os testes posteriores.

Durante as tentativas, o sujeito foi orientado a tocar o elástico na fase excêntrica a cada repetição, garantindo assim a ADM desejada no exercício. Em seguida, foi adicionada carga na barra e o sujeito foi orientado a realizar 10 repetições máximas (10-RM). Foram realizadas, no máximo três tentativas por dia, com intervalo de $05 \mathrm{~min}$ entre elas. Para novo teste, foi dado um intervalo mínimo de $48 \mathrm{~h}$.

Para o teste em ADM total, os mesmos procedimentos acima foram adotados, com a diferença que, para validar a repetição o sujeito deveria tocar a barra levemente no peito na fase excêntrica do movimento. Ambos os testes de 10-RM em diferentes amplitudes foram realizados no mesmo dia de forma randomizada e com intervalo mínimo de 15 min entre eles.

\section{Procedimentos}

Foram realizadas cinco visitas para a totalização da coleta de dados, com intervalo mínimo de três e máximo de sete dias entre elas. 
As três visitas iniciais foram compostas de familiarização dos voluntários com os procedimentos experimentais e a confiabilidade das medidas de ADM da cintura escapular e de força de resistência (10-RM) no exercício de supino reto com barra livre.

Ordenadas de forma aleatória, as demais visitas foram destinadas às condições experimental e controle. $\mathrm{Na}$ condição experimental, os voluntários realizaram o teste para medida da ADM da cintura escapular no movimento de abdução horizontal do ombro (AHO) e em seguida foram submetidos a uma rotina de alongamentos estáticos, realizada de forma aleatória para os músculos tríceps braquial e peitoral maior. Imediatamente após, aferiu-se novamente a ADM da AHO e em seguida os sujeitos realizaram os testes de 10RM no supino reto com a barra livre em duas ADM distintas (amplitude de movimento total e limitada a $90^{\circ}$ de flexão do cotovelo), distribuídas de forma aleatória.

O tempo de intervalo entre os testes de 10RM no supino reto nas diferentes angulações foi de $15 \mathrm{~min}$. e foi realizada uma nova rotina de alongamentos estáticos antes do segundo teste. O desenho experimental pode ser mais bem visualizado na Figura 2.

Adotaram-se os mesmos procedimentos durante a visita destinada à condição controle, com exceção da aplicação da rotina de alongamento. Durante o período que representou o tempo necessário para se cumprir as rotinas de alongamento (aproximadamente 4 min), os voluntários permaneceram sentados sem se movimentar.

$\mathrm{Na}$ visita experimental, os indivíduos foram submetidos ao protocolo de alongamento estático passivo, realizado de forma aleatória para os músculos peitoral maior e tríceps braquial. Os movimentos empregados foram os seguintes: (a) abdução horizontal da articulação do ombro com os cotovelos flexionados, (b) abdução da articulação do ombro em rotação externa com o cotovelo flexionado e palma da mão em posição supina. O primeiro movimento foi realizado bilateralmente e o segundo de forma unilateral.
Os indivíduos foram submetidos a duas séries de alongamento estático com $30 \mathrm{~s}$ de insistência na posição de maior desconforto suportado. O tempo de intervalo de uma série para outra, no mesmo membro, foi o tempo necessário para realizar o alongamento no membro contralateral. Para o movimento bilateral foi dado um intervalo de $30 \mathrm{~s}$ entre os alongamentos.

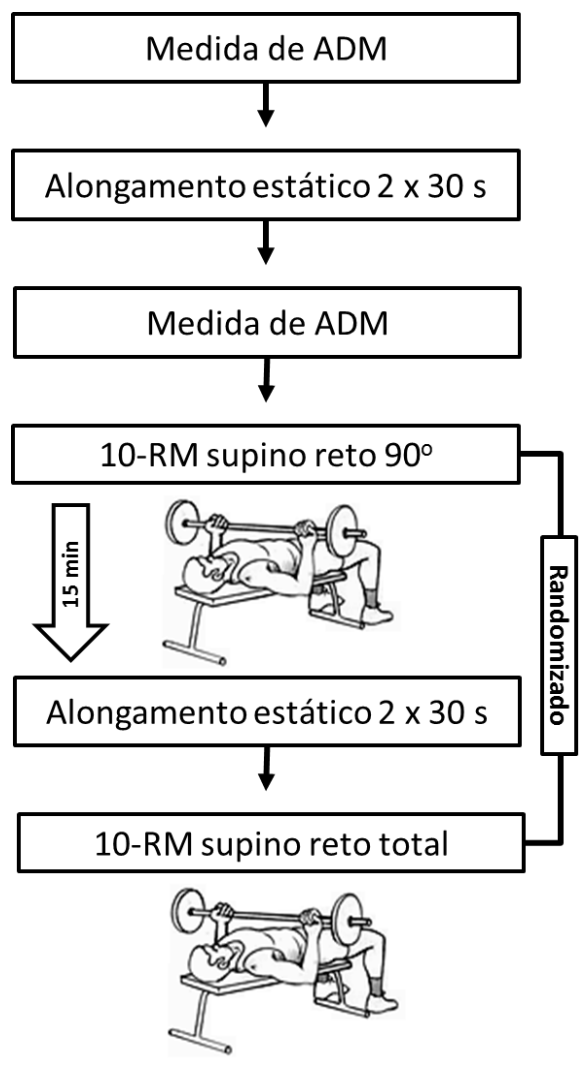

Figura 2. Desenho experimental

\section{Análise Estatística}

A estabilidade das medidas de ADM E 10RM foi determinada através do coeficiente de correlação intraclasse (CCI; método paralelo), utilizando os valores obtidos durante as duas visitas destinadas à confiabilidade das medidas.

A confiabilidade absoluta de ambas as medidas foi determinada através do cálculo do erro típico da medida (ETM). De acordo com Hopkins (2000), o ETM é determinado pela razão entre o desvio padrão das diferenças obtidas entre os pares de medida e a raiz quadrada do algarismo dois.

Uma ANOVA de duas entradas com medidas repetidas nos dois fatores (condição $\mathrm{x}$ 
amplitude de movimento) foi utilizada para comparar as diferenças entre as médias das variáveis dependentes: número máximo de repetições para carga de 10-RM e o volume total (produto da carga utilizada e número de repetições alcançadas) nas duas amplitudes testadas. Para a comparação entre as médias da ADM na AHO entre cada condição nos momentos pré vs. pós, empregou-se uma ANOVA de duas entradas com medidas repetidas nos dois fatores (condição $\mathrm{x}$ tempo). Quando observadas diferenças nos fatores principais ou na interação, estas foram determinadas através do teste post hoc de Bonferroni. Além disso, quando observado diferença significativa nas comparações, foi calculado o tamanho do efeito (d) (Cohen, 1992), a partir da diferença entre a medida pós e pré tratamento dividida pelo desvio padrão do pré para a comparação entre as médias das variáveis dependentes (número de repetições a $90^{\circ}$ e amplitude total, e ADM). Foram utilizados os pontos de corte como sugeridos por Rhea (2004), para sujeitos recreativamente treinados: efeito trivial $<0.35$; efeito pequeno $0.35-0.80$; efeito moderado 0.80-1.50; efeito grande $>1.5$.

Todas as análises foram realizadas em software estatístico comercialmente disponível (SPSS Statistics for Windows, Versão 17.0. Chicago: SPSS Inc.) e foi adotada uma significância estatística de $\alpha=0.05$.

\section{RESULTADOS}

Os valores de confiabilidade da medida (estabilidade) e ETM para as variáveis ADM do ombro, 10-RM a $90^{\circ}$ e 10-RM total estão reportados na Tabela 1.

Tabela 1.

Valores de confiabilidade e ETM absoluto e relativo para $A D M$ e 1-RM a $90^{\circ}$ e em amplitude total

\begin{tabular}{lcccc}
\hline & ETM absoluto & ETM relativo & $\mathrm{R}$ & $\mathrm{p}$ \\
\hline ADM (graus) & 5.0 & $6 \%$ & 0.959 & 0.0001 \\
$10-$ RM90 (reps) & 1.85 & $3 \%$ & 0.997 & 0.0001 \\
10-RMTot (reps) & 1.7 & $3 \%$ & 0.997 & 0.0001 \\
\hline
\end{tabular}

Pode-se observar uma confiabilidade alta para todas as medidas efetuadas. O ETM para cada uma das variáveis está descrito de forma absoluta (em função da unidade de medida) e relativa (em valores percentuais) e indica qual é o erro aleatório associado à medida em dias diferentes.

Na figura 3 encontra-se a comparação da ADM para as diferentes condições. Observou-se interação significativa $(p=0.029)$ e d de 0.44 (efeito pequeno), com aumento da ADM para o grupo que realizou alongamento. $\mathrm{O}$ aumento absoluto de aproximadamente $6^{\circ}(7 \%)$ na $\mathrm{ADM}$ para o grupo que realizou a rotina de alongamentos está além do ETM, o que reforça o poder de inferência de aumento na ADM após a rotina de alongamento estático.
Como observado na Tabela 2, não foi encontrada diferença significativa para número de repetições máximas nem no volume total (carga $\mathrm{x}$ número de repetições) nas condições $\mathrm{AL}$ e $\mathrm{CON}$ a $90^{\circ}$ e em amplitude total.

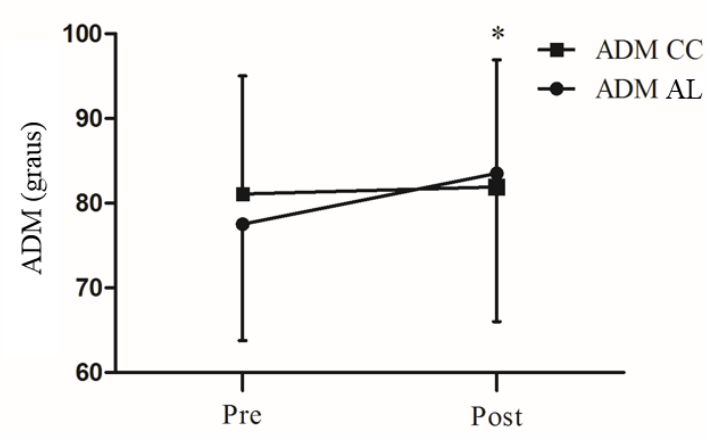

Figura 3. Comparação das médias de ADM pré e após nas condições controle (CC) e alongamento (AL). Aumento significativo $(p=0.029)$ na condição após $\mathrm{AL}$ 
Tabela 2

Valores de repetição máxima e volume total nas diferentes condiçôes e angulações

\begin{tabular}{ccccc}
\hline Condição & $\begin{array}{c}\text { RM-90 } \\
\text { (repetições) }\end{array}$ & $\begin{array}{c}\text { RM-total } \\
\text { (repetições) }\end{array}$ & VT-90 & VT-total \\
\hline Controle & $10.4 \pm 1.7$ & $11.5 \pm 1.2$ & $653.2 \pm 275.2$ & $604.3 \pm 219.5$ \\
Alongamento & $10.5 \pm 0.9$ & $10.9 \pm 1.5$ & $652.2 \pm 214.2$ & $562.2 \pm 180.1$ \\
\hline
\end{tabular}

Legenda: RM- $90^{\circ}$ - repetições máximas a $90^{\circ}$;RM-total - repetições máximas em amplitude total; VT-90 - volume total (carga x repetição) a $90^{\circ}$; VT-total - volume total (carga x repetição) em amplitude total.

\section{DISCUSSÃO}

O presente estudo analisou o efeito do alongamento estático sobre o desempenho da força de resistência (10-RM) no exercício de supino reto em diferentes amplitudes de movimento $\left(90^{\circ}\right.$ de flexão de cotovelo e amplitude total) e sobre a ADM da cintura escapular no movimento de AHO com cotovelos estendidos. O principal achado foi que rotinas de alongamento estático passivo com duas séries de $30 \mathrm{~s}$ de insistência aumentaram de forma significativa a ADM na AHO sem provocar queda no desempenho de repetições máximas ou volume total (carga $\mathrm{x}$ repetições) em diferentes angulações no exercício de supino reto.

Nossos achados reforçam o corpo de evidências que encontraram aumento na ADM sem queda significativa no desempenho após rotinas mais curtas de alongamentos estáticos (Ogura et al., 2007; Ryan et al., 2008). Além disso, outros estudos têm evidenciado a ausência de efeito deletério do alongamento estático sobre a força quando volumes menores (ex., 2 x 30 s) são empregados (Beedle et al., 2008; Endlich et al., 2009).

No estudo de Endlich et al. (2009), os autores só encontraram queda significativa na carga máxima $(\mathrm{kg})$ no teste de $10-\mathrm{RM}$ para a condição com $16 \mathrm{~min}$. de alongamento. No entanto a rotina com volume menor (8 min) não provocou queda no desempenho. De forma semelhante, Beedle, Rytter, Healy, e Ward (2008) também não encontraram queda significativa na carga $(\mathrm{kg})$ utilizada no teste de 1-RM no supino reto em homens e mulheres após três séries de $15 \mathrm{~s}$ de alongamento estático. Essa relação do volume de alongamento empregado e seus efeitos sobre o desempenho da força foi postulada por Ryan et al. (2008) como efeito dose-resposta. O autor sugere que, a medida que se aumenta o volume de alongamento, os efeitos deletérios sobre a força se tornam mais evidentes. Recentemente, Simic, Sarabon, e Markovic (2013) em uma revisão de literatura e meta análise evidenciaram a tendência de se reduzir os efeitos negativos sobre a força máxima com rotinas mais reduzidas de alongamento estático.

Com relação aos mecanismos de redução da força muscular e potência após alongamento estático, Ogura, Miyahara, Naito, Katamoto, e Aoki (2007) sugerem que dois fatores estejam envolvidos: a diminuição da rigidez da unidade músculo-tendinosa como fator mecânico e a inibição da excitabilidade dos moto neurónios alfa do SNC como fator neural. Como já evidenciado na literatura, os fatores neurais têm maior possibilidade de estarem envolvidos no efeito negativo provocado pelo alongamento estático quando volumes muito extensos são utilizados (Avela, Kyröläinen, \& Komi, 1999; Fowles, Sale, \& MacDougall, 2000; Trajano, Seitz, Nosaka, \& Blazevich, 2013). No entanto, quando rotinas menores são empregadas, parece que fatores estruturais, como a redução na rigidez passiva da unidade músculotendinosa, são os principais responsáveis pela queda no desempenho (Marshall, Cashman, \& Cheema, 2011; Mizuno, Matsumoto, \& Umemura, 2013).

Recentemente foi proposto que a relação ângulo dependente dos efeitos do alongamento sobre a força. Nesse sentido, postula-se que o aumento do comprimento de repouso do sarcômero, devido a rotinas de alongamento estático, exigiria que o músculo trabalhasse em uma maior amplitude de movimento para atingir os mesmos níveis de tensão máxima alcançados sem a rotina de alongamentos, mudando a região onde o maior número de pontes cruzadas seria formado e, 
consequentemente, a maior capacidade de produzir força (Cramer et al., 2007; Fowles et al., 2000; Herda, Cramer, Ryan, McHugh, \& Stout, 2008).

Alguns autores observaram essa relação. Weir, Tingley, e Elder (2005) encontraram uma queda significativa na contração voluntária máxima (CVM) $(7,1 \%)$ a $20^{\circ}$ de dorsiflexão (pós-teste ${ }_{1}$ ) após 05 séries de $120 \mathrm{~s}$ de alongamento estático. No entanto, quando a ADM foi aumentada para $26^{\circ}$ de dorsiflexão (Pós-teste ${ }_{2}$ ), não houve diferença nos valores de CVM pré e após alongamento. De forma semelhante, LaTorre et al. (2010) testaram o efeito de $4 \times 30 \mathrm{~s}$ de alongamentos estáticos sobre o desempenho do salto vertical partindo de diferentes angulações de flexão do joelho $\left(50^{\circ}, 70^{\circ}, 90^{\circ}\right.$ e $\left.110^{\circ}\right)$ e só encontraram queda significativa na altura do salto $(20,7 \%)$ para $50^{\circ}$ de flexão do joelho.

No presente estudo, não foi observada queda significativa no desempenho do teste de 10-RM em nenhuma das angulações testadas $\left(90^{\circ} \mathrm{e}\right.$ amplitude total). Deve-se frisar que o presente estudo utilizou um volume menor de alongamentos estáticos ( $02 \times 30 \mathrm{~s})$ para os músculos testados, o que pode explicar esse fato. Além disso, pode-se supor que as angulações utilizadas não tenham sido suficientes para promover a frouxidão esperada na unidade músculo-tendinosa. Tal fato pode ser reforçado por estudos que não encontraram queda no desempenho da força em angulações maiores (Herda et al., 2008; Nelson et al., 2001) onde, provavelmente, a unidade músculotendinosa não apresentou frouxidão ou maior complacência.

O presente estudo apresenta algumas limitações. A primeira refere-se a amostra de indivíduos do sexo masculino treinados no exercício supino reto. Pode-se sugerir que indivíduos não treinados obtenham resultados diferentes aos da presente amostra. Entretanto, Simic et al. (2013) relataram, em uma metaanálise, que os efeitos do alongamento estático sobre o desempenho da força não são influenciados pelo grau de treinamento do sujeito. Outra limitação refere-se às amplitudes de movimento articular $\left(90^{\circ}\right.$ e total) utilizadas no presente estudo. Evidências apontam para queda no desempenho da força após alongamento estático com amplitudes onde o músculo se apresenta pouco alongado (La Torre et al., 2010; Nelson et al., 2001; Weir et al., 2005). No entanto, as amplitudes de movimento articular utilizadas no presente estudo reforçam a validade externa do experimento, uma vez que representam fielmente a rotina empregada no cotidiano.

\section{CONCLUSÃO}

Com base nos resultados do presente estudo, conclui-se que rotinas de alongamento com volumes reduzidos ( 2 × $30 \mathrm{~s})$ são capazes de aumentar de forma aguda os níveis de ADM sem provocar prejuízo na força obtida através do número de repetições máximas com carga prevista para 10-RM.

Além disso, diferentemente do que se prega na literatura, a mudança na amplitude de movimento do exercício não influenciou os resultados obtidos. Deve-se frisar que tal comportamento possa ser diferente caso angulações menores (próximas à extensão de cotovelos) sejam utilizadas durante o exercício. No entanto, como na prática geral desse exercício, dificilmente se percebe amplitudes inferiores a $90^{\circ}$, pode-se recomendar que, caso haja a intenção de se aumentar agudamente a ADM da articulação do ombro previamente a exercícios de força, rotinas de alongamento estático com pequeno volume podem ser utilizadas sem oferecer risco de queda significativa no desempenho.

Os resultados do presente estudo, se confirmados em outros experimentos controlados, podem modificar na prática a maneira como se prescreve o aquecimento e/ou atividades preparatórias, aquelas realizadas antes de atividades que envolvam força ou potência. É possível que naquelas atividades onde é necessário um alto grau de flexibilidade para o seu desempenho, exercícios de alongamento realizados da maneira como utilizado no presente estudo, possam ser realizados melhorando a amplitude de movimento necessária, sem que o desempenho da força seja prejudicado. 


\section{Agradecimentos: \\ Nada a declarar.}

\section{Conflito de Interesses:}

Nada a declarar.

\section{Financiamento:}

$\mathrm{O}$ estudo foi patrocinado em parte pelo $\mathrm{CNPq}$ (Brasil), Protocolo \# 907147206996512. PSCG é bolsista de produtividade (PQ) do CNPq.

\section{REFERÊNCIAS}

Avela, J., Kyröläinen, H., \& Komi, P. V. (1999). Altered reflex sensitivity after repeated and prolonged passive muscle stretching. Journal of Applied Physiology, 86(4), 1283-1291.

Bacurau, R. F. P., Monteiro, G. A., Ugrinowitsch, C., Tricoli, V., Cabral, L. F., \& Aoki, M. S. (2009). Acute effect of a ballistic and a static stretching exercise bout on flexibility and maximal strength. Journal of Strength and Conditioning Research, 23(1), 304-308. http://doi.org/10.1519/JSC.0b013e3181874d5 5

Beedle, B., Rytter, S. J., Healy, R. C., \& Ward, T. R. (2008). Pretesting static and dynamic stretching does not affect maximal strength. Journal of Strength and Conditioning Research, 22(6), $1838-1843$. http://doi.org/10.1519/JSC.0b013e3181821bc 9

Bradley, P. S., Olsen, P. D., \& Portas, M. D. (2007). The effect of static, ballistic, and proprioceptive neuromuscular facilitation stretching on vertical jump performance. Journal of Strength and Conditioning Research, 21(1), 223-226. http://doi.org/10.1519/R-21046.1

Cohen, J. (1992). A power primer. Psychological Bulletin, 112(1), 155-159. http://doi.org/10.1037/0033-2909.112.1.155

Cramer, J. T., Beck, T. W., Housh, T. J., Massey, L. L., Marek, S. M., Danglemeier, S., ... Egan, A. D. (2007). Acute effects of static stretching on characteristics of the isokinetic angle - torque relationship, surface electromyography, and mechanomyography. Journal of Sports Sciences, 25(6), 687-698. http://doi.org/10.1080/02640410600818416

Endlich, P. W., Farina, G. R., Dambroz, C., Gonçalves, W. L. S., Moysés, M. R., Mill, J. G., \& Abreu, G. R. de. (2009). Acute effects of static stretching in dynamic force performance in young men. Revista Brasileira de Medicina do Esporte,

15(3), 200-203.
http://doi.org/10.1590/S151786922009000300007

Fowles, J. R., Sale, D. G., \& MacDougall, J. D. (2000). Reduced strength after passive stretch of the human plantarflexors. Journal of Applied Physiology, 89(3), 1179-1188.

Herda, T. J., Cramer, J. T., Ryan, E. D., McHugh, M. P., \& Stout, J. R. (2008). Acute effects of static versus dynamic stretching on isometric peak torque, electromyography, and mechanomyography of the biceps femoris muscle. Journal of Strength and Conditioning Research, 22(3), 809-817. http://doi.org/10.1519/JSC.0b013e31816a82e c

Hopkins, W. G. (2000). Measures of reliability in sports medicine and science. Sports Medicine, 30(1), 1-15. http://doi.org/10.2165/00007256-20003001000001

La Torre, A., Castagna, C., Gervasoni, E., Cè, E., Rampichini, S., Ferrarin, M., \& Merati, G. (2010). Acute effects of static stretching on squat jump performance at different knee starting angles. Journal of Strength and Conditioning Research, 24(3), 687-694. http://doi.org/10.1519/JSC.0b013e3181c7b44 3

Marshall, P. W. M., Cashman, A., \& Cheema, B. S. (2011). A randomized controlled trial for the effect of passive stretching on measures of hamstring extensibility, passive stiffness, strength, and stretch tolerance. Journal of Science and Medicine in Sport, 14(6), 535-540. http://doi.org/10.1016/j.jsams.2011.05.003

Mizuno, T., Matsumoto, M., \& Umemura, Y. (2013). Decrements in stiffness are restored within 10 min. International Journal of Sports Medicine, 34(6), 484-490. http://doi.org/10.1055/s0032-1327655

Nelson, A. G., Guillory, I. K., Cornwell, C., \& Kokkonen, J. (2001). Inhibition of maximal voluntary isokinetic torque production following stretching is velocity-specific. Journal of Strength and Conditioning Research, 15(2), 241-246. http://doi.org/10.1519/00124278200105000-00014

Ogura, Y., Miyahara, Y., Naito, H., Katamoto, S., \& Aoki, J. (2007). Duration of static stretching influences muscle force production in hamstring muscles. Journal of Strength and Conditioning Research, 21(3), 788-792. http://doi.org/10.1519/R-18785.1

Perrier, E. T., Pavol, M. J., \& Hoffman, M. A. (2011). The acute effects of a warm-up including static or dynamic stretching on countermovement jump height, reaction time, and flexibility. Journal of Strength and Conditioning Research, 25(7), 1925-1931. http://doi.org/10.1519/JSC.0b013e3181e7395 
Rhea, M. R. (2004). Determining the magnitude of treatment effects in strength training research through the use of the effect size. Journal of Strength and Conditioning Research, 18(4), 918-920. http://doi.org/10.1519/14403.1

Rubini, E. C., Costa, A. L. L., \& Gomes, P. S. C. (2007). The effects of stretching on strength performance. Sports Medicine, 37(3), 213-224. http://doi.org/10.2165/00007256-20073703000003

Ryan, E. D., Beck, T. W., Herda, T. J., Hull, H. R., Hartman, M. J., Stout, J. R., \& Cramer, J. T. (2008). Do practical durations of stretching alter muscle strength? A dose-response study. Medicine and Science in Sports and Exercise, $40(8)$,

$1529-1537$. http://doi.org/10.1249/MSS.0b013e31817242 eb

Simic, L., Sarabon, N., \& Markovic, G. (2013). Does pre-exercise static stretching inhibit maximal muscular performance? A meta-analytical review. Scandinavian Journal of Medicine \& Science in Sports, 23(2), 131-148. http://doi.org/10.1111/j.16000838.2012.01444.x

Trajano, G. S., Seitz, L., Nosaka, K., \& Blazevich, A. J. (2013). Contribution of central vs. peripheral factors to the force loss induced by passive stretch of the human plantar flexors. Journal of Applied Physiology, 115(2), 212-218. http://doi.org/10.1152/japplphysiol.00333.20 13

Weir, D. E., Tingley, J., \& Elder, G. C. B. (2005). Acute passive stretching alters the mechanical properties of human plantar flexors and the optimal angle for maximal voluntary contraction. European Journal of Applied Physiology, 93(5-6), 614-623. http://doi.org/10.1007/s00421-004-1265-4 